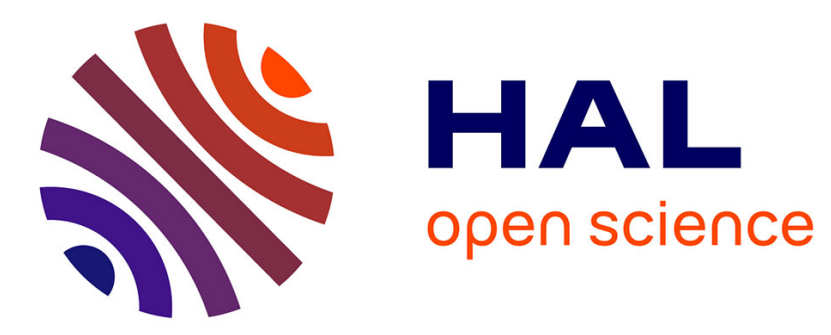

\title{
Onde de gravité dans un liquide avec film superficiel et diffusion
}

Michel Dudeck, Roger Prud'Homme

\section{To cite this version:}

Michel Dudeck, Roger Prud'Homme. Onde de gravité dans un liquide avec film superficiel et diffusion. International Journal of Heat and Mass Transfer, 1978. hal-02004798

\section{HAL Id: hal-02004798 \\ https://hal.science/hal-02004798}

Submitted on 9 Apr 2020

HAL is a multi-disciplinary open access archive for the deposit and dissemination of scientific research documents, whether they are published or not. The documents may come from teaching and research institutions in France or abroad, or from public or private research centers.
L'archive ouverte pluridisciplinaire HAL, est destinée au dépôt et à la diffusion de documents scientifiques de niveau recherche, publiés ou non, émanant des établissements d'enseignement et de recherche français ou étrangers, des laboratoires publics ou privés. 


\title{
ONDE DE GRAVITE DANS UN LIQUIDE AVEC FILM SUPERFICIEL ET DIFFUSION
}

\author{
M. Dudeck ct R. Prud'homme
}

Laboratoire d'Aérothermique du CNRS. 4 ter, route des Gardes 92190, Meudon, Fr

\section{RESUME}

La propagation d'une onde de gravité de faible amplitude dans un liquide recouvert par un film est présentée en tenant compte d'un phénomène d'adsorption-désorption à l'interface liquide-film. Deux types d'onde liés respectivement au phénomène de diffusion sont étudiés : le premier type conduit à un seul mode de propagation et le second à deux modes compatibles partiellement avec les conditions aux limites suivant le signe de la quantité $\eta_{D}^{* 2}-2 \pi \eta_{r}^{*} B^{1 / 2}$.

\section{NOMENCLATURE}

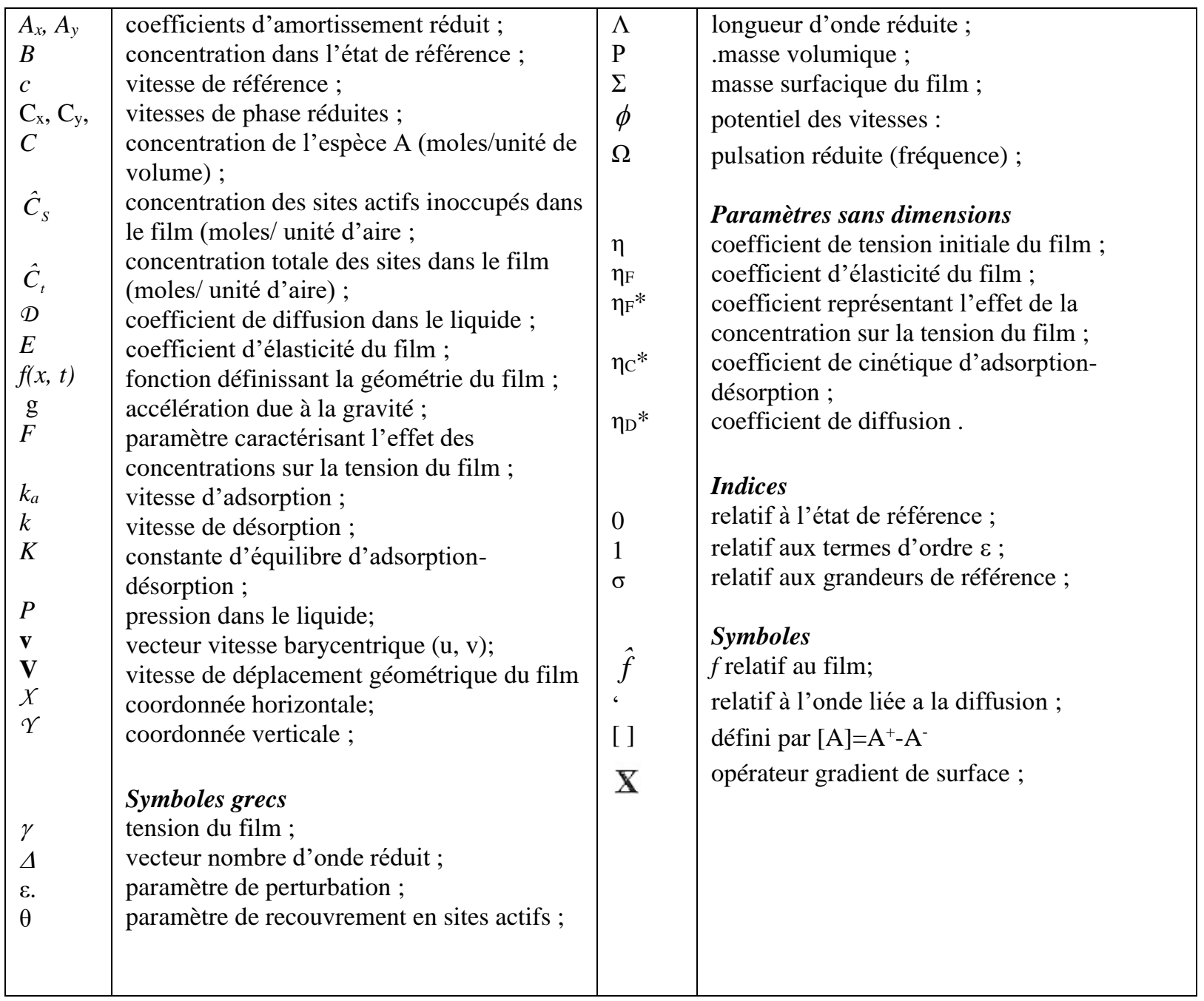




\section{INTRODUCTION}

La PROPAGATION d'une onde de gravité de faible amplitude dans un liquide est modifiée par la présence d'un film superficiel. Ces modifications sont étudiées ici en présence d'un phénomène d'adsorption-désorption à l'interface liquide-film.

Dans le modèle étudié, le liquide admet une profondeur infinie et comprend deux constituants A et B. Le film superficiel est considéré comme un milieu sans épaisseur caractérisé par des grandeurs internes telles que sa vitesse, sa masse surfacique, sa concentration et sa tension. La possibilité d'un décollement du film sur le liquide est supposée exclue et l'étude des conditions de rupture n'est pas envisagée.

L'échange entre le liquide et le film s'effectue par un processus d'adsorptiondésorption du constituant A seul. Cet échange produit une modification de la concentration dans le film, modifie sa tension, et provoque un phénomène de diffusion dans le liquide.

Le gaz situé au-dessus du film n'intervient que par sa pression, supposée constante et uniforme. Aucun échange n'est envisagé avec ce gaz.

L'ensemble liquide-film est soumis â une onde de gravité de faible amplitude: On admet une pro propagation bidimensionnelle et isotherme de l'onde et un comportement de fluide parfait pour le liquide (Fig. 1).

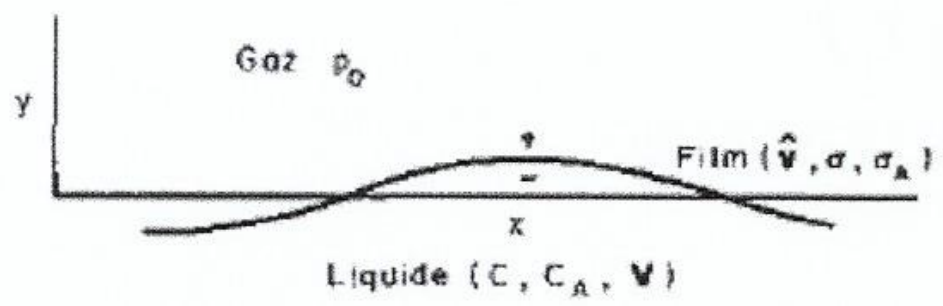

Fig. 1. Milieu liquide-film-gaz

Les équations de bilan, la condition d'échange et les conditions aux limites sont linéarisées à partir d'un paramètre e caractérisant l'amplitude de la perturbation de la surface libre.

La solution du système d'équations ainsi obtenu est recherchée sous la forme d'une combinaison de deux ondes. La première est associée nu phénomène de diffusion dans le liquide et la seconde est associée au champ de vitesse du liquide. Deux équations de dispersion sont obtenues. elles relient la vitesse complexe de phase et la pulsation de chacune des ondes aux différents paramètres du milieu. Les solutions de ces équations sont discutées principalement en fonction de deux paramètres sans dimensions $\eta_{C}^{*}$ et $\eta_{D}^{*}$ liés à l'échange entre le film et le liquide Ces paramètres caractérisent respectivement la cinétique d'adsorption-désorption et la diffusion dans le liquide. L'étude du vecteur nombre d'onde permet de déterminer parmi les solutions obtenues celles compatibles avec un fond infini au repos et un amortissement suivant le sens de la propagation horizontale de l'onde.

L'évolution d'un film à la surface d'un liquide composé d'un seul constituant et soumis à une onde de gravité a été étudiée antérieurement pour différentes lois de comportement du film (film élastique ou viscoélastique, film a tension constante) et pour différents types d'échanges (film imperméable ou poreux) [1,2]. Les études effectuées précédemment par d'autres auteurs et portant sur I'influence de la diffusion [3,4] admettent généralement l'équilibre entre la concentration dans le film et la concentration dans le liquide 
au voisinage du film. Dans notre modèle, nous avons admis au contraire une adsorptiondésorption hors d'équilibre, une tension du film fonction de la masse surfacique totale et de la masse surfacique de l'espèce A et. de plus, la possibilité d'un glissement du film sur le liquide.

\section{MISE EN EQUATION}

Trois équations de bilan sont nécessaires pour caractériser l'évolution isotherme du film, ce sont les équations de bilan de la masse globale, de bilan de la masse de l'espèce A et de bilan de la quantité de mouvement.

Pour a masse globale, on obtient [5]: $\mathbf{X}$

$$
\frac{\partial \sigma}{\partial t}+\sigma(\mathbf{X} \cdot \hat{\mathbf{v}}-\hat{\mathbf{v}} \cdot \mathbf{X} \mathbf{1})+[\rho(\mathbf{v}-\mathbf{V})] \cdot \boldsymbol{\xi}=0
$$

avec: $\bar{d} / d t$ dérivée particulaire égale à $(\partial / \partial t)+\hat{v} \cdot \nabla ; \boldsymbol{X}$ opérateur « diabolo » défini par $(\mathbf{1}-\boldsymbol{\xi} \otimes \boldsymbol{\xi})-(\mathbf{V} \cdot \boldsymbol{\xi}) \boldsymbol{\xi}$ et, $\boldsymbol{\xi}$ vecteur unitaire normal l'interface.

Le film n'échange pas de matière avec le gaz, le terme entre crochets représente donc la masse de l'espèce $A$ adsorbée par unité d'aire et de temps.

Aucune réaction chimique n'est envisagée entre le film et le liquide.

La masse d'espèce $A$ absorbée par la réaction

s'exprime par son taux de production

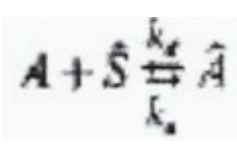

$$
\dot{\Omega}=\mathcal{M}_{A}\left(k_{a} C \hat{C}_{S}-k_{D} \hat{C}\right)
$$

$\hat{S}$ représente un site actif et $\hat{A}$ représente l'espèce $A$ adsorbée; $k_{a}$ et $k_{d}$ sont respectivement la vitesse d'adsorption et la vitesse de désorption de l'espèce $A$; $C$ est le nombre de moles de l'espèce $A$ par unité de volume dans le liquide, $\hat{C}_{S}$ est le nombre de sites actifs (multiplié par le nombre d'Avogadro par unité d'aire et $\mathcal{M}_{\mathrm{A}}$ est la masse molaire de l'espèce $A$.

Le flux de masse échangé entre le film et le liquide est donc:

$$
\begin{aligned}
& \dot{\Omega}=[\rho(\mathbf{v}-\mathbf{V})] \cdot \xi=\rho\left(\mathbf{v}_{\mathbf{1}}^{-} \cdot \xi-V_{\xi}\right) \\
& =\mathcal{M}_{A}\left(k_{a} C \hat{C}_{S}-k_{D} \hat{C}\right)
\end{aligned}
$$

Le nombre de sites occupés par l'espèce $A$ adsorbée, plus le nombre de sites actifs inoccupés est égal au nombre total de sites qui sera supposé constant par unité d'aire, soit :

$$
\hat{C}+\hat{C}_{S}=\hat{C}_{t}
$$

Deux cas limites seront envisagés l'équilibre et le figeage d'adsorption-désorption.

L'équilibre est défini par : 


$$
\frac{\hat{C}}{C\left(\hat{C}_{t}-\hat{C}\right)}=\frac{k_{a}}{k_{D}}=K(T)
$$

Afin de conserver un débit d'adsorption non nul, l'équilibre doit être considéré comme un cas limite où les deux conditions suivantes sont vérifiées :

$$
\begin{aligned}
& 1-\frac{\hat{C}}{K(T) C\left(\hat{C}_{t}-\hat{C}\right)} \rightarrow 0 \\
& \mathcal{M}_{A} k_{a} C \hat{C}_{S} \rightarrow \infty
\end{aligned}
$$
soit :

Le deuxième cas limite est celui du figeage pour lequel le débit d'adsorption est nul,

$$
\rho\left(\mathbf{v}_{1}^{-} \cdot \xi-V_{\xi}\right)=0
$$

Le figeage impose les conditions:

$$
\begin{aligned}
& 1-\frac{\hat{C}}{K(T) C\left(\hat{C}_{t}-\hat{C}\right)} \text { fini, } \\
& \mathcal{M}_{A} k_{a} C \hat{C}_{S} \rightarrow 0
\end{aligned}
$$

La condition (10) est analogue à celle du film imperméable pour lequel les projections sur la normale $\xi$ de la vitesse barycentrique du liquide et de la vitesse géométrique du film sont égales.

En admettant que la diffusion dans le liquide est traduite par la loi de Fick, l'équation de bilan de la masse de l'espèce $A$ dans le film s'écrit :

$$
\frac{\hat{d} \hat{C}}{d t}+\hat{C}(\mathbf{X} \cdot \hat{\mathbf{v}}-\hat{\mathbf{v}} \cdot \mathbf{X} \mathbf{1})+[\mathscr{D V} C-C(\mathbf{v}-\mathbf{V})] \cdot \xi=0
$$

où $\mathcal{D}$ représente le coefficient de diffusion.

On suppose qu'aucun effet de viscosité n'apparaît dans le liquide [3] et que le tenseur des tensions de surface est tel que :

$$
\gamma=\gamma(\mathbf{1}-\xi \otimes \xi)
$$

L'équation de bilan de la quantité de mouvement du film, compte tenu de l'équation de bilan de masse s'écrit :

$$
\sigma \frac{\mathrm{d} \hat{\mathbf{v}}}{\mathrm{d} t}-\mathbf{X} \gamma+[p \mathbf{1}+\rho(\mathbf{v}-\hat{\mathbf{v}}) \otimes(\mathbf{v}-\mathbf{V})] \cdot \xi=\sigma \mathbf{g}
$$

où $p$ représente le champ dc pression dans le liquide. Cette équation de bilan tient compte de l'inertie du film.

La tension $\gamma$ du film est reliée à la niasse surfacique globale $\sigma$ et à la concentration surfacique $\hat{C}$ en espèce $A$ par la loi de comportement : 


$$
\gamma=\gamma_{0}-\frac{E}{\sigma_{0}}\left(\sigma-\sigma_{0}\right)-\frac{F}{\hat{C}_{0}}\left(\hat{C}-\hat{C}_{0}\right)
$$

où le coefficient $E$ traduit le caractère élastique du film et le coefficient $F$ exprime l'influence de la concentration en $A$ dans le film sur la tension.

Le liquide est considéré comme un fluide partait, incompressible et irrotationnel, les équations classiques de Bernoulli et de Laplace sont donc vérifiées.

$$
\frac{\partial \phi}{\partial t}+\frac{v^{2}}{2}+\frac{p}{\rho}+g y=C(t)
$$

et

$$
\Delta \phi=0
$$

où $\phi$ représente le potentiel des vitesses tel que $\mathbf{v}=\nabla \phi$.

L'équation de bilan de la masse de l'espèce $A$ dans le liquide s'écrit

$$
\frac{\partial C}{\partial t}+\mathbf{v} \cdot \nabla C=\mathscr{D} \Delta C
$$

\section{EQUATIONS LINEAIRES}

Dans l'état de référence (indice 0) le liquide au repos est recouvert du film qui est alors plan et immobile, il y a équilibre chimique et équilibre mécanique entre le film et le liquide. L'équilibre d'adsorption-désorption entraîne

$$
\frac{\hat{C}_{0}}{C_{0}\left(\hat{C}_{t}-\hat{C}_{0}\right)}=K(T)
$$

L'équilibre mécanique impose :

$$
\hat{\mathbf{v}}_{0}=\mathbf{0} \text { et } \mathbf{v}_{0}=\mathbf{0}
$$

Lorsque l'ensemble liquide-film est soumis à une perturbation de faible amplitude $\varepsilon$, la modification de la forme du film entraine une variation de sa tension et de sa concentration en espèce $A$. Il y a alors échange dc masse entre le film et le liquide par un phénomène d'adsorption- désorption.

Tous les termes figurant dans les équations sont développés suivant les puissances croissantes du paramètre de perturbation $\varepsilon$, toute grandeur $\psi$ admettant un développement de la forme

$$
\Psi(\mathrm{x}, \mathrm{y}, \mathrm{t})=\Psi_{0}(\mathrm{x}, \mathrm{y})+\varepsilon \Psi_{1}(\mathrm{x}, \mathrm{y}, \mathrm{t})+\mathrm{O}\left(\varepsilon^{2}\right)
$$

Lorsque les termes d'ordre $\varepsilon$ sont seuls retenus, on obtient l'ensemble d'équations suivant :

surface du liquide

$$
y=\varepsilon \mathrm{f}(x, t)
$$

bilan de masse globale dans le film 


$$
\sigma_{1, t}+\sigma_{0} \hat{u}_{1, x}=\rho_{0}\left(v_{1}^{-}-f_{1}{ }^{\prime}\right) \quad \text { en } y=0
$$

bilan de concentrations en espèce $A$ dans le film, la diffusion de l'espèce $A$ dans le film est négligée,

$$
\hat{C}_{1, t}+\hat{C}_{0} \hat{u}_{1, t}=C_{0}\left(v_{1}^{-}-f_{1}{ }^{\prime}\right)_{-} D C_{1, y} \quad \text { en } y=0 ;
$$

bilan de quantité de mouvement du film suivant l'axe des $\mathrm{x}$,

$$
\sigma_{0} \hat{u}_{1, t}+\frac{E}{\sigma_{0}} \sigma_{1, x}+\frac{F}{\hat{C}_{0}} \hat{C}_{1, x}+f_{1, x} \sigma_{0} g
$$

bilan de quantité de mouvement du film suivant l'axe des y,

$$
\text { en } y=0 \text {; }
$$

$$
\sigma_{0} \hat{v}_{1, t}-\gamma_{0} f_{, x x}+\sigma_{1} g=p_{1}^{-} \quad \text { en } y=0 \text {, }
$$

avec

$$
p=p_{0}+\sigma_{0} g+\varepsilon p_{1}
$$

adsorption-désorption

$$
\rho_{0}\left(v_{1}^{-}-f_{1}^{\prime}\right)=M_{A} k_{d} \hat{C}_{0}\left(\frac{C_{1}}{C_{0}}+\frac{\hat{C}_{S 1}}{\hat{C}_{S 0}}-\frac{\hat{C}_{1}}{\hat{C}_{0}}\right) \quad \text { en } y=0 ;
$$

bilan dc masse de l'espèce $A$ dans le liquide où les termes de convection sont négligés car d'ordre 2 suivant $\varepsilon$;

$$
C_{1, t}-D\left(C_{1, x x}+C_{1, y y}\right)=0 \quad \text { en } y=0 ;
$$

bilan des sites

$$
\hat{C}_{1}+\hat{C}_{S 1}=0
$$

incompressibilité du liquide

$$
\phi_{1, x x}+\phi_{, y y}=0
$$

équation de Bernoulli

$$
\phi_{1, t}+g f+\frac{p_{1}^{-}}{\rho_{0}}=0 \quad \text { en } y=0 ;
$$

condition de compatibilité géométrique

$$
\hat{v}_{1}=f_{, t} \quad \text { en } \mathrm{y}=0 \text {; }
$$

conditions d'amortissement de la perturbation pour la variable $y$ tendant vers $-\infty$

$$
C_{1}=0 \text { et } \mathbf{v}_{1}=0
$$

La solution du problème couplé de transfert de masse et de quantité de mouvement à l'ordre un est alors complètement déterminé par les équations (20)-(33). 


\section{EQUATIONS D’ONDE}

Les équations de propagation de l'onde mécanique (30) et de l'onde de diffusion (28) excluent toute solution monochromatique pour les grandeurs $\phi_{1}$ et $C_{1}$ ou toute solution de la forme

$$
\left[\begin{array}{l}
\phi_{1} \\
C_{1}
\end{array}\right]=\left[\begin{array}{l}
\bar{\phi}_{1} \exp (m y) \\
\bar{C}_{1} \exp (m y)
\end{array}\right] \exp [i(k x-\omega t)]
$$

La solution est recherchée à partir de la propagation de deux ondes : une onde mécanique et une onde de diffusion, soit :

$$
\begin{aligned}
& \phi_{1}=\bar{\phi} \exp \left[i\left(\mathbf{K} \cdot \mathbf{r}_{-} \omega t\right)\right] \\
& C_{1}=\bar{C} \exp \left[i\left(\mathbf{K} \cdot \mathbf{r}_{-} \omega t\right)\right]
\end{aligned}
$$

avec

$$
\mathbf{K}=\left[\begin{array}{l}
K_{x} \\
K_{y}
\end{array}\right], \mathbf{K}^{\prime}=\left[\begin{array}{l}
K^{\prime}{ }_{x} \\
K^{\prime}{ }_{y}
\end{array}\right] \text { et } \mathbf{r}=\left[\begin{array}{l}
x \\
y
\end{array}\right]
$$

Les quantités liées au film $\sigma_{1}, f_{1}, \hat{u}_{1}$ et $\hat{C}_{1}$ apparaissent alors comme des combinaisons linéaires de ces deux ondes, soit :

$$
\left[\begin{array}{l}
\sigma_{1} \\
f \\
\hat{u}_{1} \\
\hat{C}_{1}
\end{array}\right]=\left[\begin{array}{c}
\bar{\sigma} \\
\bar{f} \\
\bar{u} \\
\bar{c}
\end{array}\right]\left[i\left(\mathbf{K} \cdot \mathbf{r}_{-} \omega t\right)\right]+\left[\begin{array}{c}
\bar{\Sigma} \\
\bar{F} \\
\bar{U} \\
\bar{C}
\end{array}\right]\left[i\left(\mathbf{K}^{\prime} \cdot \mathbf{r}_{-} \omega^{\prime} t\right)\right]
$$

Cette solution est compatible avec le système d'équations linéarisées si deux équations d'onde sont vérifiées. Les équations (23)-(2?) et (29)-(32) permettent d'établir les deux équations suivantes:

$$
\begin{aligned}
& \left(1-\Omega^{2}+\eta_{\gamma}^{2} \Omega^{2} \Delta_{x}^{2}+i \frac{\Omega}{\Delta_{\gamma}}\right)\left\{-\eta_{F}^{2} \Delta_{x}+\frac{1}{B \Delta_{x}}\left(1+i \frac{\eta_{C}^{* 2}}{\Omega}\right)-\eta_{C}^{* 2} \Delta_{x}\left[i \frac{\eta_{C}^{* 2}}{\Omega}\left(\frac{1}{B}-1\right)+\frac{1}{B}\right]\right\} \\
& +\Delta_{x}\left[\frac{1}{B}+i \frac{\eta_{C}^{* 2}}{\Omega}\left(\frac{1}{B}-1\right)+\frac{\eta_{C}^{* 2}}{\Delta_{\gamma}}\right]=0 \\
& -\eta_{F}^{* 2} \Delta_{x}^{\prime 2}\left[1+i \Omega^{\prime} \Delta_{F}^{\prime} \frac{\eta_{D}^{* 2}}{\eta_{C}^{* 2}}+\Omega^{\prime 2} B\left(\eta_{\gamma}^{2} \Delta_{x}^{\prime 2}-1\right)\right] \\
& +\left(1+\eta_{D}^{* 2} \Delta_{F}^{\prime}\right)\left[-\Delta_{x}^{\prime 2} \eta_{F}^{* 2}\left(1-\Omega^{\prime 2}+\eta_{\gamma}^{2} \Omega^{\prime 2} \Delta_{x}^{\prime 2}\right)+\Delta_{x}^{\prime 2}+\Omega^{\prime 2}\left(\eta_{\gamma}^{2} \Delta_{x}^{\prime 2}-1\right)\right]
\end{aligned}
$$


Les équations (26 et (24) conduisent aux relations suivantes entre les valeurs d'onde complexes :

$$
\begin{aligned}
& \Delta_{x}^{2}+\Delta_{y}^{2}=0 \\
& \Delta_{x}^{\prime}{ }^{2}+\Delta_{y}^{\prime}{ }^{2}=\frac{1}{B \eta_{D}^{* 2}\left(1-\bar{\theta}_{0}\right) \Omega^{\prime}}
\end{aligned}
$$

Les quantités sans dimensions introduites dans les équations (38)-(41) sont les suivantes:

\section{Variables sans dimensions}

$\Omega=\omega / \omega_{\sigma}\left(\Omega^{\prime}=\omega / \omega_{C}\right)$ pulsation réduite où $\omega_{\sigma}$ est une pulsation de référence

$\Delta_{x}=K_{x} c_{\omega} / \omega,\left(\Delta_{x}^{\prime}=K_{x}^{\prime} c_{\omega} / \omega^{\prime}\right)$ nombre d'onde suivant $x$ où $c_{\omega}$ est une vitesse de référence associée au film,

$\Delta_{y}=K_{y} c_{\omega} / \omega,\left(\Delta_{y}^{\prime}=K_{y}^{\prime} c_{\omega} / \omega^{\prime}\right)$ nombre d'onde suivant $y$;

la pulsation de référence $\omega_{\sigma} \phi t$ la vitesse de référence sont définies par

\section{Paramètres sans dimensions}

$\eta_{\gamma}=c_{\gamma} / c_{\sigma}$ tension initiale du film $c_{\gamma}^{2}=\gamma_{0} \omega^{2} / \rho_{0} \theta$,

$\eta_{E}=c_{E} / c_{\sigma}$ caractéristique d'influence de la masse surfacique sur la tension du film,

$\eta_{F}^{*}=c_{F} / c_{\sigma}$ caractéristique d'influence de la masse surfacique de l'espèce $\mathrm{A}$ sur la tension $\mathrm{du}$ film $c_{F}^{2}=F C_{0} / \rho_{0} \hat{C}_{0}$,

$\eta_{C}^{*}=\left[\mathcal{M}_{A} k_{d} C_{0} /\left(1-\hat{\theta}_{0}\right) \rho_{0} \omega_{\sigma}\right]^{1 / 2}$ caractéristique de la cinétique d'adsorption-désorption,

$\eta_{D}^{*}=\left[D C_{0} / c_{\sigma}\left(1-\hat{\theta}_{0}\right) \hat{C}_{0}\right]^{1 / 2}$ caractéristique de la diffusion dans le liquide,

$\mathrm{B}=B=\rho_{0} \hat{C}_{0} / \sigma_{0} C_{0}$.

La quantité $\hat{\theta}_{0}$ est définie par le rapport $\hat{C}_{0} / \hat{C}_{t}$ ce terme représente le taux de recouvrement des sites actifs. L'équation (20) re!ie $\hat{\theta}_{0}$ à la concentration $C_{0}$ de I'espèce $A$ dans le liquide. Le coefficient $B$ représente le rapport de deux longueurs caractéristiques, l'une associée à la masse globale et l'autre à la masse de l'espèce $A$.

L'équation d'onde pour un film imperméable [2] s'obtient, à partir de (38) et (40), avec

$$
\eta_{C}^{*}=\eta_{D}^{*}=\eta_{F}^{*}=0
$$

soit,

$$
\left(1-\eta_{E}^{2} \Delta_{x}^{2}\right)\left(1-\Omega^{2}+\frac{i \Omega}{\Delta_{F}}-\eta_{\gamma}^{2} \Omega^{2} \Delta_{x}^{2}\right)+\Delta_{x}^{2}=0
$$

pour l'onde mécanique.

Les équations (39) et (41) conduisent alors à une propagation horizontale pour l'onde de diffusion (Section 52). 


\section{RESU LTATS ET DISCUSSION}

Les équations d'onde (38)-(41) ont été résolues numériquement par la méthode de Jenkins-Traub. Les solutions recherchées correspondent à une propagation dirigée suivant les valeurs croissances de $x$ et à un amortissement de l'onde dans le sens de la propagation.

Les vitesses de phase et les coefficients d'amortissement de l'onde sont définis par:

$$
\begin{aligned}
& \Delta_{x}=\frac{1}{C_{x}}+i A_{x}, \Delta_{y}=\frac{1}{C_{y}}+i A_{y} \\
& \Delta_{x}^{\prime}=\frac{1}{C^{\prime}{ }_{x}}+i A^{\prime}{ }_{x}, \Delta_{y}^{\prime}=\frac{1}{C^{\prime}}+i A^{\prime}{ }_{y}
\end{aligned}
$$

L'évolution de ces grandeurs est principalement étudiée en fonction de la pulsation réduite $\Omega$ (et $\Omega^{\prime}$ ) ou de la longueur d'onde réduite $\Lambda=C / \Omega$ (et $\Lambda^{\prime}=C^{\prime} / \Omega^{\prime}$ ).

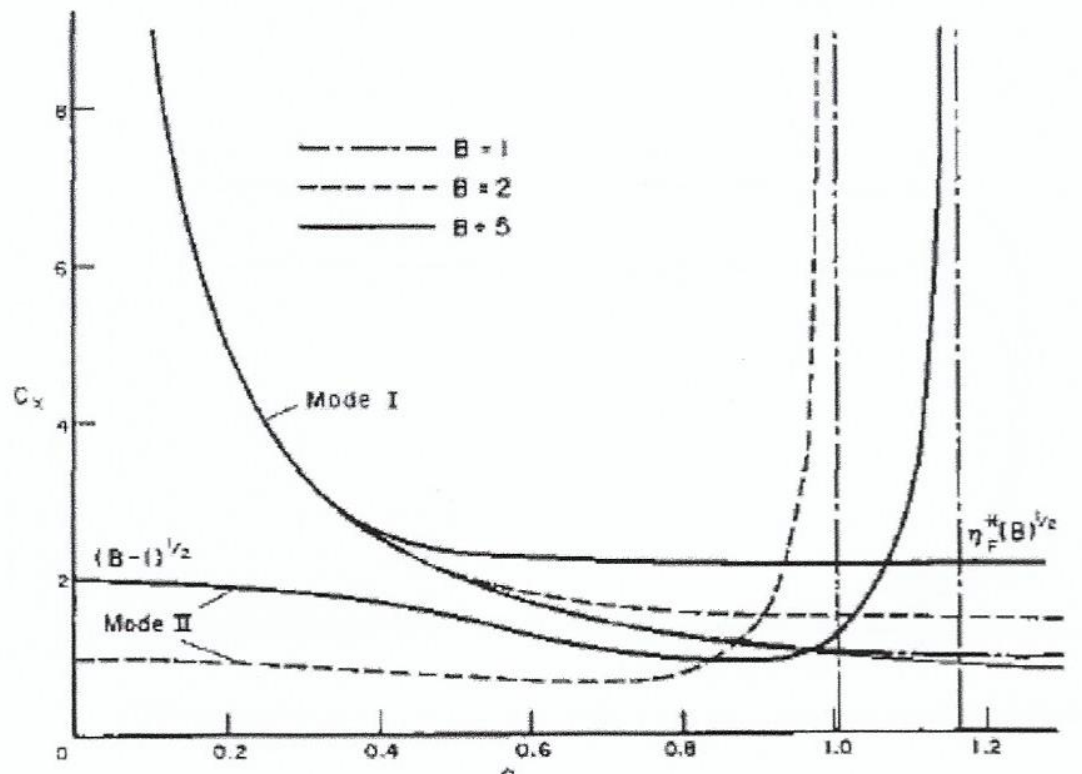

Fig. 2. Vitesse de phase en fonction de la pulsation trait fin : surface libre $C_{x}=\Omega^{-1} ; \eta_{E}=\eta_{\gamma}, \eta_{F}^{*}=\eta_{C}^{*}=1$.

\subsection{Equation d'onde mécanique}

La première équation d'onde est établie à partir de l'équation (38) et (40) et est compatible avec un amortissement dc l'onde pour y tendant vers - z, on obtient :

$$
\begin{aligned}
& \Delta_{x}^{3}\left\{-\eta_{F}^{2}\left(1-\Omega^{2}\right)+\frac{1}{B}+\eta_{\gamma}^{2} \frac{\Omega^{2}}{B}+i\left[\left(\frac{1}{B}-1\right) \frac{\eta_{C}^{* 2}}{\Omega}+\eta_{\gamma}^{2} \eta_{C}^{2} \frac{\Omega}{B}\right]\right\} \\
& +\Delta_{x}^{2}\left(\eta_{F}^{2} \Omega+i \eta_{C}^{*_{2}}\right)+\Delta_{x}\left(\frac{1-\Omega^{2}}{B}\right)\left(1+i \frac{\eta_{C}^{* 2}}{\Omega}\right)-\frac{\Omega}{B}-i \frac{\eta_{C}^{* 2}}{B}=0
\end{aligned}
$$

$\operatorname{avec} \Delta_{y}=-i \Delta_{x}$. 
Cette équation conduit à un ou deux modes de propagation dans la direction des $x$ croissants Pour un coefficient $B$ supérieur à l'unité deux modes de propagation apparaissent (modes I et II, Fig. 2). Pour les faibles fréquences, la vitesse de phase du mode I correspond à celle des oscillations du liquide avec surface libre $C_{x}=\Omega^{-1}$. Pour les grandes pulsations de ce mode, la vitesse $C_{x}$, tend vers la valeur limite $\eta_{F} *(B)^{1 / 2}$. Le deuxième mode, qui n'existe que pour B supérieur à l'unité, correspond à une vitesse égale à $(B-1)^{1 / 2}$ pour les faibles pulsations et admet une vitesse infinie pour une pulsation finie. Les Fig. 3 et 4 présentent les variations du coefficient d'amortissement associé aux modes I et II en fonction de la longueur d'onde réduite : seul le mode I correspond à urne onde amortie pour les valeurs croissantes de $x$.

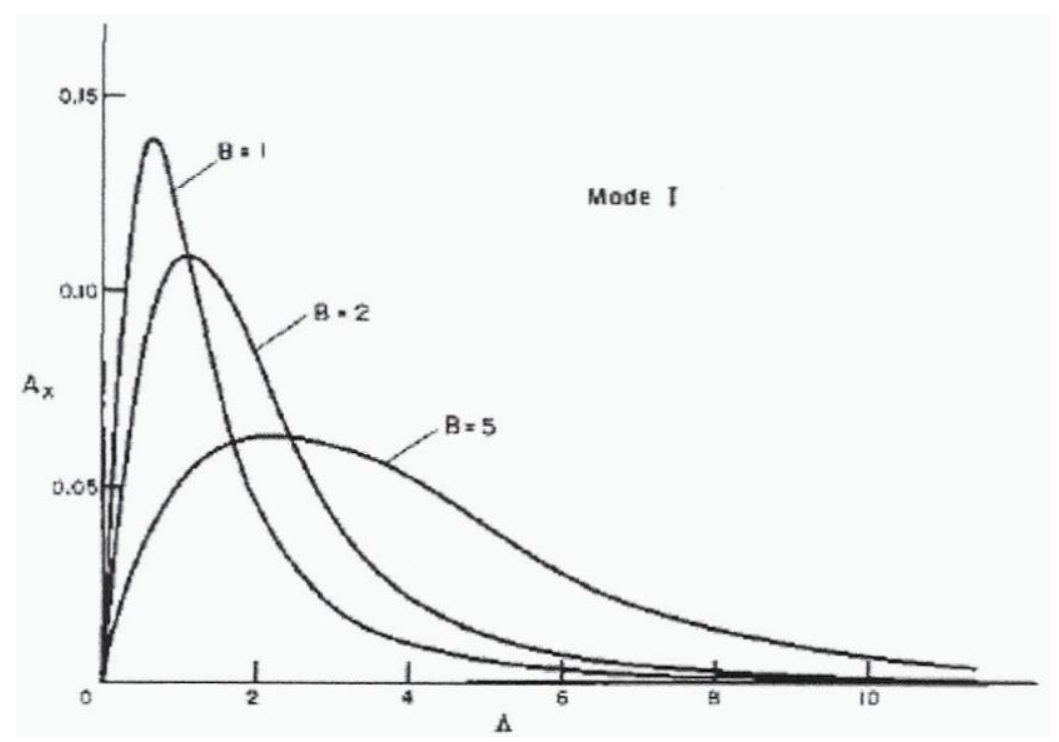

Fig. 3 Coefficient d'amortissement réduit en fonction de la longueur d'onde réduite (mode I).

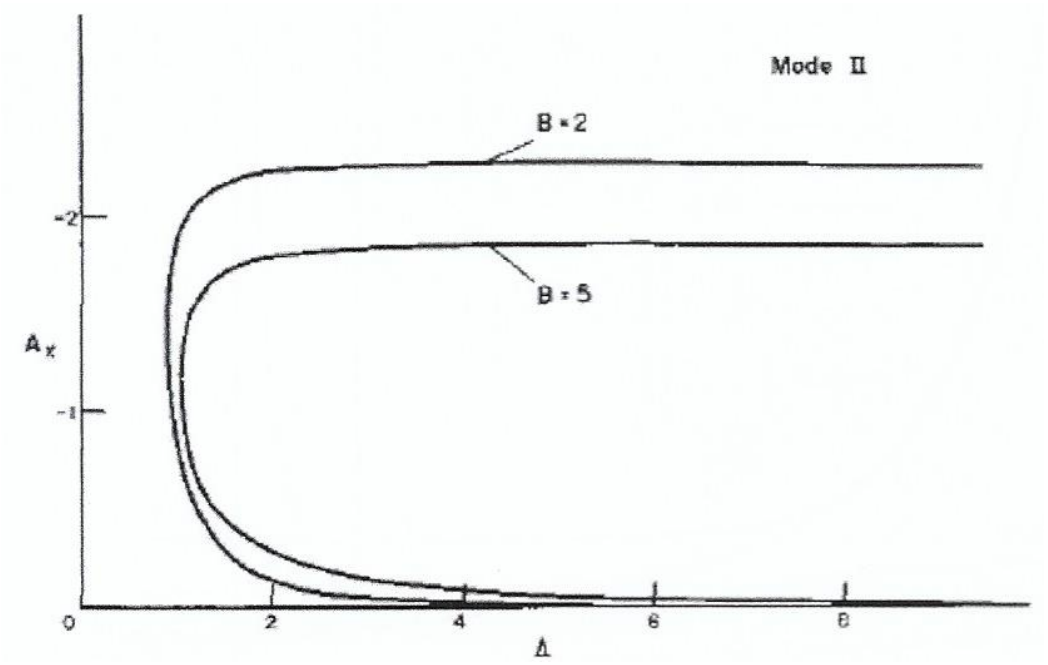

Fig 4. Coefficient d'amortissement réduit en fonction de la longueur d'onde réduite (mode II). 

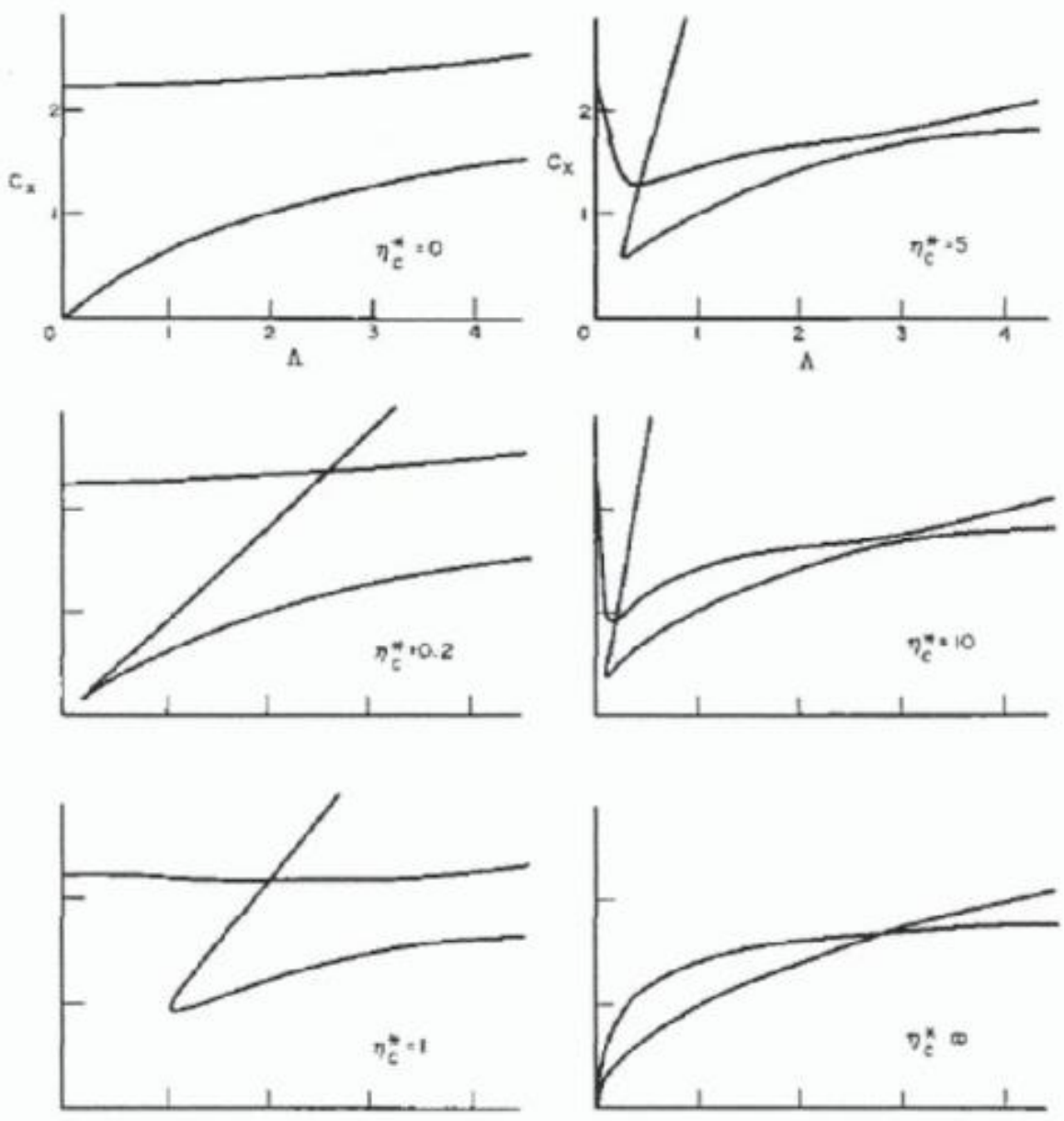

Fig. 5. Influence de la cinétique d'adsorption-désorption sur la vitesse de phase réduite pour $\eta_{C}{ }^{*}$ variant de 0 à l'infini, $\eta_{E}=\eta_{F}=0, \eta_{F}{ }^{*}=1$ et $B=5$.

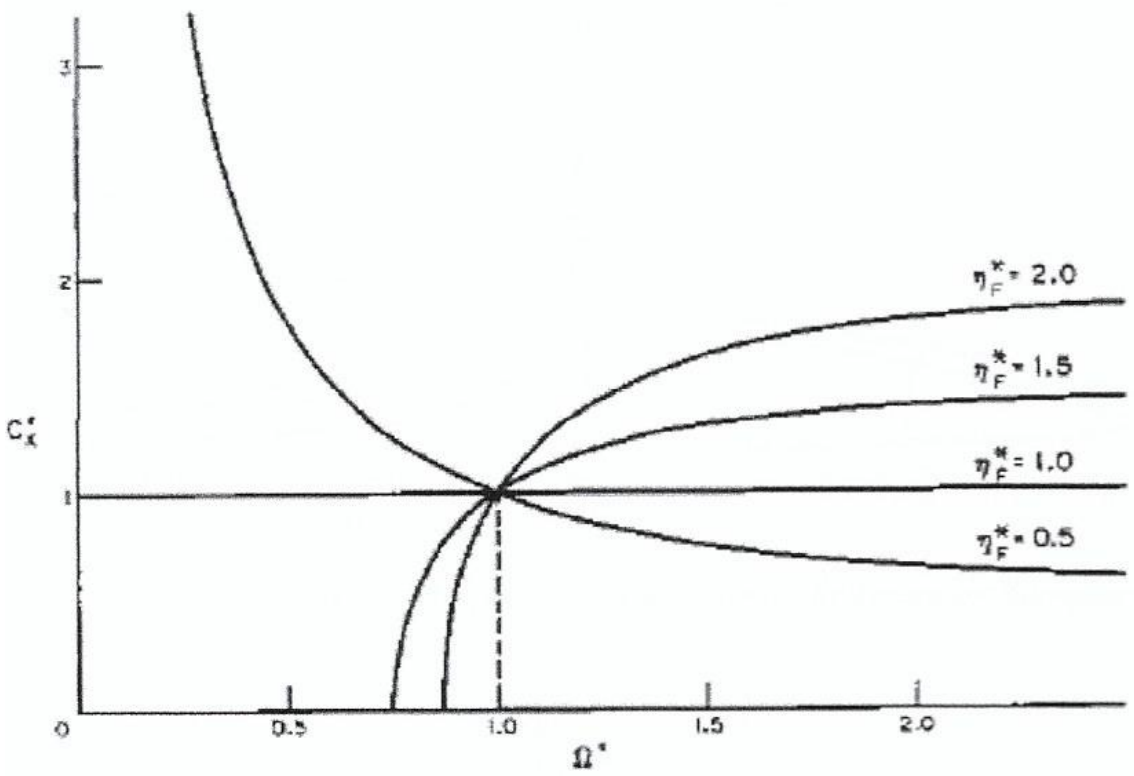

Fig.6. Vitesse de phase en fonction.de la pulsation pour $\eta_{D} * \rightarrow 0$. 
La cinétique d'adsorption-désorption est traduite par le paramètre sans dimension $\eta_{C}{ }^{*}$. A l'équilibre, c'est à-dire pour $\eta_{C} *$ tendant vers l'infini, on obtient une première solution $C x=\Omega^{-1}$ et une deuxième solution définie pour $B>1$ :

$$
C_{x}=\frac{2(B-1)}{\Omega+\left[\Omega^{2}+4(B-1)\right]^{1 / 2}}
$$

Ces deux solutions correspondent à un amortissement nul suivant l'axe des $x$.

L'évolution de la vitesse de phase entre les deux cas limites (adsorption-désorption figée et à l'équilibre) est présentée sur 1a Fig. 5 pour $\eta_{\gamma}$ nul.

\subsection{Equation d'onde de diffusion}

Lorsque le paramètre $\eta$ est. nul, les équations (39)et (41) conduisent à la deuxième équation d'onde suivante :

$$
\begin{aligned}
& \Delta_{F}^{\prime 3}\left(-\eta_{C}^{* 2}+i \eta_{F}^{*_{2}} \Omega^{\prime}\right)+\Delta_{F}^{\prime 2}\left(\eta_{F}^{* 2}-\eta_{F}^{* 2} B \Omega^{\prime 2}-1\right) \eta_{C}^{* 2} \Omega^{\prime} \\
& +\Delta_{y}^{\prime}\left[-\Omega^{3} \eta_{D}^{* 2} \eta_{C}^{* 2}-\frac{\eta_{F}^{* 2} \Omega}{B\left(1-\hat{\theta}_{0}\right)}+i \frac{\eta_{C}^{* 2}}{B\left(1-\hat{\theta}_{0}\right)}\right] \\
& -\Omega^{\prime 3} \eta_{C}^{* 2}+i \frac{\eta_{C}^{* 2}}{B \eta_{D}^{* 2}\left(1-\hat{\theta}_{0}\right)}\left(-\eta_{F}^{* 2}+\eta_{F}^{* 2} B \Omega^{\prime 2}+1\right)=0
\end{aligned}
$$

L’influence de la diffusion sur la propagation associée a été principalement étudiée.

Pour une diffusion nulle dans le liquide $\left(\eta_{D^{*}}=0\right)$, la vitesse de propagation est nulle suivant l'axe des $y$ et l'équation (39) devient :

$$
\Delta_{x}^{\prime 2}=\frac{\Omega^{\prime 2}}{1-\eta_{F}^{* 2} B \Omega^{\prime 2}}
$$

avec une vitesse de propagation nulle suivant l'axe des $y$. Les variations de la vitesse de propagation $C^{\prime}{ }_{x}$ de l'onde suivant l'axe des $x$ sont présentées sur la Fig. 6 en fonction de la pulsation réduite $\Omega$ ' pour $B=1$ et pour différentes valeurs du paramètre $\eta_{F}{ }^{*}$. Pour $\eta_{F}{ }^{*}$ plus grand que l'unité, le mode de propagation obtenu n'est défini que pour $\Omega^{\prime} \geq\left[\left(\eta_{F}^{* 2}-1\right) / \eta_{F}^{* 2} B\right]^{1 / 2}$ Toutes les courbes obtenues passent par le point défini par $\Omega^{\prime}=B^{-1 / 2}, C^{\prime}{ }_{x}=B^{1 / 2}\left(\Lambda^{\prime}=B\right)$, ces solutions sont non amorties suivant $x$.

La solution de la deuxième équation d'onde dépend du signe de la quantité $\eta_{D} *^{2}-2 \eta_{F} *(B)^{1 / 2}$. Trois modes de propagation sont obtenus.

Le mode I est tel que $A_{y}^{\prime}$ est négatif pour $\eta_{D} *^{2} \leq 2 \eta_{F}^{*}(B)^{1 / 2}$. Pour $\eta_{D}{ }^{2}>2 \eta_{F}^{*}(B)^{1 / 2}$, l'onde n'est amortie pour y $\longrightarrow-\infty$ que pour $\Omega^{\prime}<\Omega^{\prime}{ }_{1}$. Pour ce mode, la vitesse de propagation $C_{y}^{\prime}$ est toujours négative. Dans la plan $\left(C_{y}^{\prime}, \Omega^{\prime}\right)$ deux branches sont obtenues pour $\eta_{D}{ }^{2}<2$ $\eta_{F} *(B)^{1 / 2}$ (une seule correspond à un amortissement suivant $\mathrm{x}$ ) et une seule branche apparait pour $\eta_{D^{* 2}}>2 \eta_{F}{ }^{*}(B)^{1 / 2}$ (solution a suivant $x$ ), Dans le plan $\left(C^{\prime}{ }_{y}, \Omega^{\prime}\right.$ ) deux branches apparaissent pour $\eta_{D}{ }^{2}<2 \eta_{F}^{*}(B)^{1 / 2}$, seule la branche associée aux faibles pulsations est amortie suivant les $x$ croissants. Pour $\eta_{D}{ }^{2} \geq 2 \eta_{F} *(B)^{1 / 2}$, seule subsiste la branche associée à une onde amortie.

Le mode 2 n'est jamais compatible avec la condition aux limites pour $\mathrm{y} \longrightarrow$ l'infini. Ce mode est associé une onde amortie pour les $x$ croissants sauf pour $\Omega$ 'voisin de zéro. 
Le mode 3 n'est amorti suivant $y$ que pour $\Omega>\Omega^{\prime}{ }_{2}$. Cc mode admet deux branches dans le plan $\left(C_{y}^{\prime}, \Omega^{\prime}\right)$ seulement pour $\eta_{D}{ }^{2}<2 \eta_{F}{ }^{*}(B)^{1 / 2}$. Pour $\Omega^{\prime}$ tendant vers zéro, $C_{y}^{\prime}$ tend vers la valeur $\eta_{D}{ }^{2} /\left(\eta_{F}{ }^{2}-1\right)$ et l'onde est toujours amortie suivant $x$.

L'évolution des grandeurs $C^{\prime}{ }_{x}, C^{\prime}{ }_{y}, A^{\prime}{ }_{x}$ et $A^{\prime}{ }_{y}$ est représentée en fonction de $\Omega$ ' sur les Figs.7et 8 , pour $\eta_{D} *^{2}>2 \eta_{F}^{*}(B)^{1 / 2}$.

Pour $\eta_{D} *$ tendant vers l'infini, un seul mode de propagation est obtenu, il est défini par:

$$
\Delta_{x}^{\prime 2}=\frac{\Omega^{\prime 2}}{1-i \Omega^{\prime} \frac{\eta_{F}^{* 2}}{\eta_{C}^{* 2}}}
$$

Pour ce mode de propagation de l'onde, $A^{\prime}{ }_{x}$ est positif et $A^{\prime}{ }_{y}$ négatif.
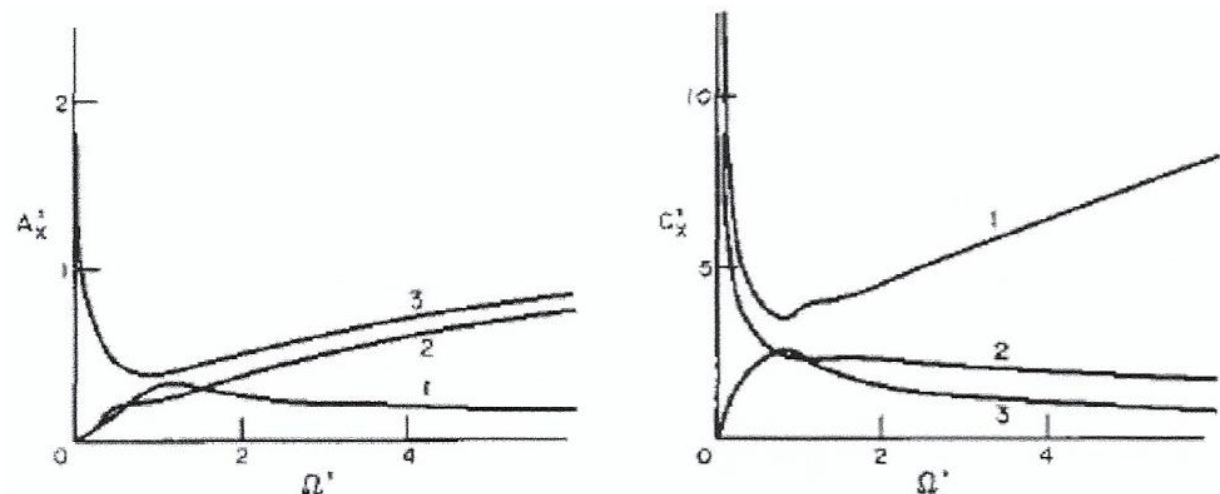

Fig. 7. Caractéristiques du vecteur d'onde suivant l'axe des $x$,
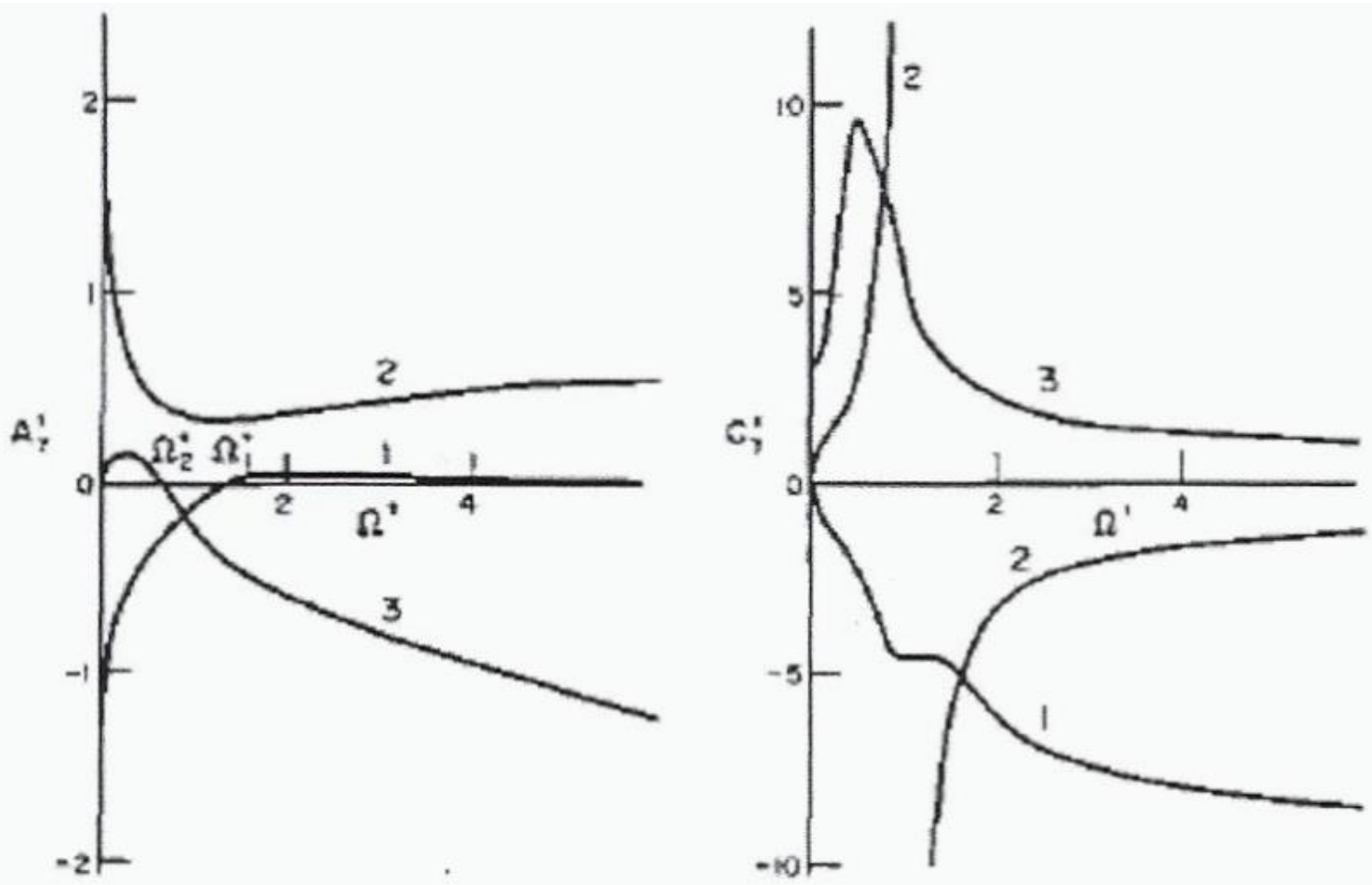

Fig. 8. Caractéristiques du vecteur d'onde suivant l'axe des $y$, 


\section{6, CONCLUSIONS}

Nous avons présenté l'effet du couplage entre un liquide et un film superficiel sur la propagation d'une onde de gravité dc faible amplitude. Deux liaisons interviennent entre le film et le liquide, l'une d'origine mécanique et l'autre provenant de l'adsorption-désorption. Deux équations d'onde sont établies et discutées. La première équation, liée à l'onde d'origine mécanique, permet de mettre en évidence un seul mode de propagation compatible avec un amortissement suivant les $x$ croissants et la condition aux limites pour $y$ tendant vers $-\infty$. La deuxième équation liée à l'onde d'origine dissipative, admet deux modes compatibles partiellement avec ces condition aux limites suivant le signe de la quantité $\eta_{D} *^{2}-2 \eta_{F} *(B)^{1 / 2}$. L'influence de la cinétique d'adsorption sur la première équation d'onde et l'influence dc la diffusion sur la deuxième équation d'onde ont été particulièrement étudiées.

Deux extensions possibles de ce travail sont envisagées. L'une fait intervenir d'autres natures de couplage liquide-film-gaz (ex : évaporation du film, réactions chimiques ou viscosité), l'autre utilise une approche non linéaire du problème de propagation.

\section{RKEFERENCES}

1. M. Dudeck et R. Prud'homme; Persistence of a film on a liquid in the presence of disturbance propagation, J. Appl. Phys. 48 (I) 59-66 (1977).

2. M. Dudeck et R. Prud'homme, Equation de dispersion d'un fluide avec film dc surface. Rapport 77-1 du Laboratoire d'Aérothermique du CN.R.S. (1977).

3. R.E. Zelazo et J. R. Mekher, Dynamic interactions of mono-molecular films with imposed electric fields. Physics Fluids 17, 1 (1974).

4. R. S. Hansen et J A. Man, Propagation characteristics of capillary ripples. I The theory of velocity dispersion and amplitude attenuation of plane capillary waves of viscoelastic films, J. Appl Phys. 35, 1(1974)

5. M. Barrère et R Prud'homme. Equations Fondamentales de l'Aérothermochimie, Chapitre 3, p. 110. Masson, Paris, 1973. 